\title{
L'assemblage comme forme d'innovation
}

La fabrication d'un selfie stick à Shenzhen

\section{Clément Renaud}

\section{(2) OpenEdition}

Journals

Édition électronique

URL : https://journals.openedition.org/tc/8476

DOI : $10.4000 /$ tc. 8476

ISSN : 1952-420X

Éditeur

Éditions de l'EHESS

\section{Édition imprimée}

Date de publication : 6 juin 2017

Pagination : 100-115

ISBN : 978-2-7132-2707-3

ISSN : 0248-6016

Référence électronique

Clément Renaud, "L'assemblage comme forme d'innovation », Techniques \& Culture [En ligne], 67 |

2017, mis en ligne le 06 juin 2019, consulté le 29 septembre 2022. URL : http://

journals.openedition.org/tc/8476; DOI : https://doi.org/10.4000/tc.8476 

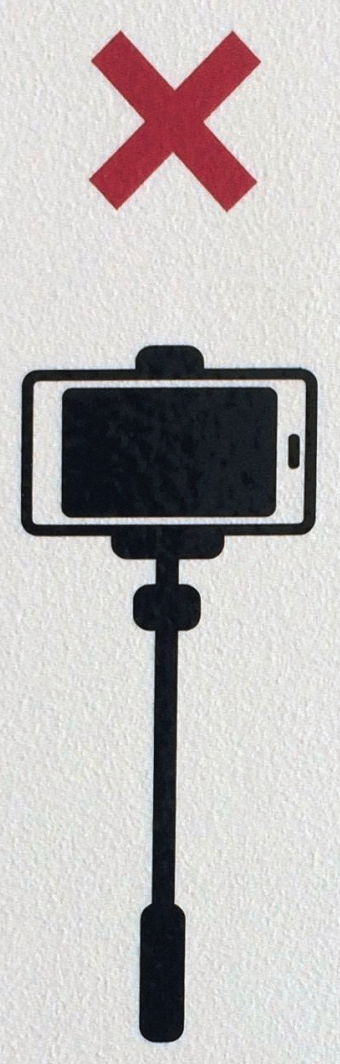

No

selfie sticks 


\title{
L'assemblage comme forme d'innovation
}

\author{
La fabrication d'un selfie stick à Shenzhen
}

L'être humain est ainsi fait qu'il lui est impossible de voir son propre visage. Chez l'humain, la représentation de soi passe donc souvent par l'intermédiaire de diverses prothèses et instruments, comme le miroir. Depuis peu de temps, la combinaison de la photographie et des systèmes de publication en ligne a vu naître un genre nouveau d'autoportrait : le « selfie ». Pris à bout de bras, le selfie est une photo mettant en scène son auteur, avec la plupart du temps l'intention de diffuser ensuite l'image sur les réseaux sociaux. L'auteur de selfies se heurte cependant à un autre problème physique : la longueur limitée de son bras ne lui autorise pas le recul nécessaire pour élargir le cadre de sa photo et en montrer le contexte (un paysage, un événement, un groupe, etc.) Afin de remédier à ce problème, une invention permet aujourd'hui d'empêcher que le visage de celui qui se prend ainsi en photo, lui-même, vienne obstruer le cadre : c'est le selfie stick ou «perche à selfie ». Composé d'une pince à téléphone montée sur un tube, ce dispositif vient ajouter à l'anatomie humaine les quelques dizaines de centimètres manquant pour réaliser une photo pouvant comprendre au besoin le décor, plusieurs personnages et le photographe lui-même. En quelques années, cette prothèse a gagné rapidement en popularité. Elle est devenue un incontournable pour un bon nombre des auteurs de selfies.

Nous allons ici nous plonger dans le trajet peu commun de cette invention, depuis sa conception jusqu'à sa fabrication et son usage. Pour ce faire, je me suis rendu en Chine à Shenzhen où la plupart de ces objets sont construits, vendus et souvent même conçus. La ville de Shenzhen est un des hauts lieux de la production de composants électroniques dans le monde et à ce titre offre un emplacement idéal pour observer les processus liés à la fabrication industrielle, et l'importance qu'ils peuvent prendre dans la définition de nouveaux objets technologiques. 
Dans ce papier, j'explore les procédés de fabrication industrielle dans les petites usines autour de Shenzhen et du Delta de la Rivière des Perles grâce à l'exemple du selfie stick. Je souhaite notamment mettre à jour le rôle particulier de l'assemblage dans les processus d'innovation. Cette article débute par un bref regard historique sur l'apparition de la perche à selfie et montre comment cet objet peut constituer un cas intéressant pour l'étude de l'apparition de technologies. Ensuite, je dresse une revue des éléments de la littérature concernant les relations entre innovation et fabrication. Après avoir présenté la ville de Shenzhen et ma méthodologie, je donne un compte rendu d'observations de terrain avant d'en discuter les résultats, en mobilisant notamment l'idée d'assemblage. Je conclue sur l'importance de la fabrication industrielle comme sujet central d'études pour les acteurs des sciences humaines s'intéressant aux techniques, et son importance capitale dans la construction de notre rapport aux technologies et plus largement au travail, à l'environnement et au monde.

\section{Le selfie stick : d'abord inutile, puis utilisable et enfin utilisé}

Avec l'apparition de la photographie, puis du téléphone mobile, le selfie s'est imposé depuis quelques décennies comme une figure de style moderne de la pratique de l'autoportrait (Lecoq-Ramond et al. 2016). Montrant la joue gauche plutôt que la droite (Bruno et al. 2015), le selfie constitue aujourd'hui une pratique mondialisée témoignant de l'importance de la représentation de soi par les technologies (Rettberg 2014). La technologie du téléphone portable a permis une diffusion massive de cette pratique du portrait qui souffre pourtant d'un défaut majeur : le visage du sujet photographié bouche le champ. Afin de pouvoir restituer l'auto-portraitiste dans son contexte, il est nécessaire de « prendre du recul » pour s'inscrire dans le cadre plus vaste des environs.

Afin de posséder le recul nécessaire, le téléphone doit être affublé d'une seconde prothèse, un bâton souvent télescopique permettant le déclenchement à distance d'une photo au cadre plus large. Cette invention connaît aujourd'hui un succès important sous le nom de selfie stick. Avérée en photographie dès $1926^{1}$, sa première mention a comme origine la pratique japonaise du chindogu consistant à imaginer des gadgets « utiles mais inutilisables ». Kenji Kawakami, maître de cet art particulier, publie dans son livre 101 Unuseless Japanese Inventions (2005) une description précise du selfie stick. Kawakami décrit cet objet incongru d'après un brevet déposé vingt ans plus tôt par des chercheurs de la compagnie Minolta, intitulé Telescopic extender for supporting compact camera (Ueda \& Mima 1985). À ce jour, pas moins d'une dizaine d'inventeurs de multiples pays ont déposé des brevets pour en clamer la paternité.

Le 3 mars 2014, la vedette américaine Ellen DeGeneres devait transformer à jamais le destin de cette invention en écrivant ces mots : "Si seulement le bras de Bradley était plus long. Meilleure photo jamais faite aux oscars ${ }^{2} »$, accompagnés d'une photographie immortalisant une brochette de 12 stars sur le grill de la prestigieuse soirée des Oscars 2014. Diffusé 
sur Twitter, le cliché soulève instantanément les soupçons des internautes : comment autant de personnes célèbres peuvent-elles entrer dans une seule et même image ? Comment un bras humain peut-il être aussi long ? Quelques heures plus tard, l'utilisation du selfie stick était révélée au monde, devenant ainsi un des plus grands hits des ventes de Noël 2014. « Nous ne pouvions tout simplement pas les garder en stock ${ }^{3} »$, explique le propriétaire d'un magasin de photo à Boulder, Colorado aux États-Unis. Malin, le fondateur du site selfiestick.co.uk raconte : "J'ai acheté le nom de domaine sur le champ, confectionné un site pendant la pause de midi et j'ai commencé à envoyer des selfie sticks depuis la Chine ${ }^{4} »$.

D'abord populaire en Asie, le selfie stick a rapidement gagné les marchés du monde entier. Avec pour destination préférée les grands sites touristiques, il permet aujourd'hui aux voyageurs solitaires de rapporter de précieuses preuves de leurs péripéties. Pourtant, les nombreux cas de blessures ou de décès dû aux selfie sticks (foudroiement, chute dans des ravins, combats rapprochés ${ }^{5} .$. ) ont rapidement amené les régulateurs à se prononcer sur l'usage du fameux bâton. Classiquement, les interdictions frappèrent d'abord les stades ${ }^{6}$, obligeant parfois à la mise en place de mesures de sécurité spéciales ${ }^{7}$ devant se généraliser à différents lieux publics, comme les musées notamment.

\section{Le design, l'innovation-conception et la question de la fabrication}

Inventeurs, conflits de paternité, succès sur le marché, problèmes de régulations... nous sommes bien ici devant un cas d'innovation tel que décrit dans les livres scolaires. Trait d'union entre le monde des réseaux sociaux en ligne et celui, plus off line, des loisirs et des vacances, la « perche à selfie » ne témoigne pas vraiment de l'immense complexité technique que l'on prête à la plupart des innovations de notre temps. Dans sa plus simple version, il est constitué d'une poignée, d'un tube télescopique et d'un connecteur pour téléphones (cable mini-jack ou puce Bluetooth selon les versions). Cette invention se présente avant tout comme un composé intégrant des briques techniques préexistantes, et dont l'apport principal est une application à un usage bien défini.

Les théories de l'innovation font généralement la place belle aux questions de conception et aux concepteurs. Alors que le modèle traditionnel du brevet cherchait à identifier précisément un auteur, des approches plus récentes se sont intéressées au rôle de l'utilisateur dans le processus de conception, partie prenante de la redéfinition d'une invention par les usages (von Hippel 2006). Le concept d'innovation ouverte proposé par Chesbrough (2003) a permis de mieux comprendre comment les firmes de R\&D pouvaient bénéficier des processus d'échanges avec des acteurs extérieurs à leurs laboratoires de recherche. Les recherches autour du design participatif ont quant à elles cherché à comprendre le rôle des utilisateurs dans le design lui-même (Simonsen \& Robertson 2013), défini comme un processus itératif de 


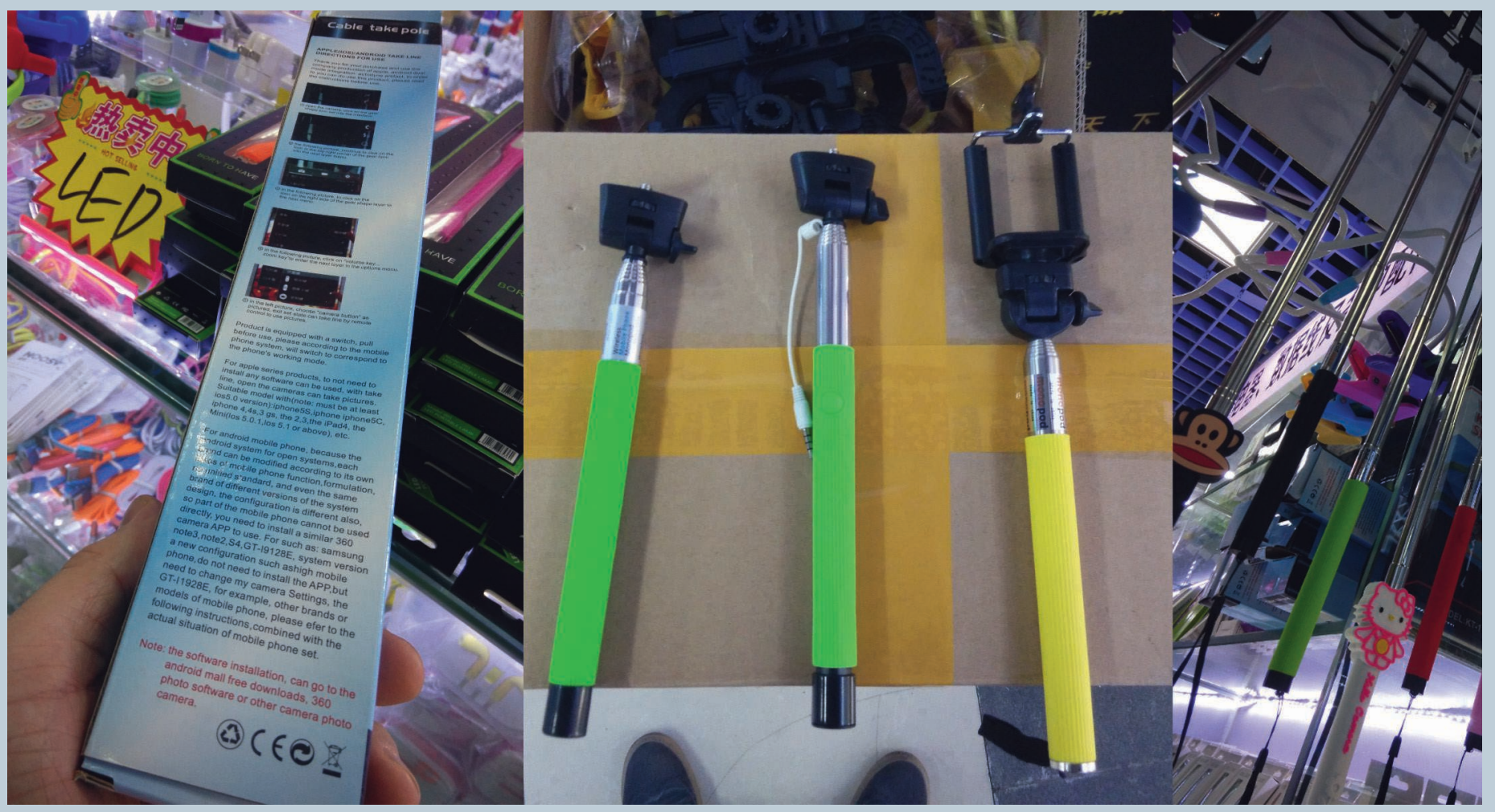

Différents modèles de selfie stick dans le quartier de Huaqiangbei 
conception. Le décentrement de la figure de l'inventeur vers un ensemble d'acteurs participant à la conception d'une innovation technologique est donc un processus largement débattu dans la littérature. Edgerton (1998) a également montré comment les récits historiques entourant les techniques se concentrent la plupart du temps sur la question de l'innovation plutôt que sur celle des technologies, menant notamment à une représentation disproportionnée du rôle de l'inventeur dans l'avènement historique de telle ou telle technique.

Le machinisme incroyable déployé par l'industrie moderne pour équiper le monde en outils et objets technologiques est largement et abondamment critiquée en tant que phénomène, notamment dans son impact sur l'écriture (Ong 2002) ou l'organisation politique du monde (Stiegler 2008, Morozov 2014). Pourtant, on trouve à ma connaissance relativement peu de travaux de terrains présentant une approche détaillée des lieux, organisations et dynamiques concernant la production manufacturière des objets et infrastructures utilisés par les technologies numériques.

\section{Ia ville de Shenzhen, du shanzhai au mouvement maker}

Trop peu de recherches ont tenté de construire un appareil critique des processus manufacturiers mis en œuvre par les dynamiques d'innovation en les mettant en rapport avec les stratégies de conception, de productions et d'usages qui leur sont associées. La plupart des travaux qui vont en ce sens ont été menés en Chine. Ce pays offre en effet un exemple fascinant et un terrain pratiquement infini pour l'étude des phénomènes de mise en ouvre et fabrication des technologies les plus variées. Avec la politique de développement du « Made in China » mené par le pays depuis sa réouverture au marché mondial au début des années quatre-vingt, chaque pièce ou produit contenant de l'électronique semble avoir transité par la Chine à un moment de son existence. De la production au recyclage des déchets électroniques, les usines chinoises jouent un rôle crucial et encore mal compris dans les processus d'innovation technologique qui se jouent dans le monde depuis 30 ans. Le programme Made in China 2025 récemment annoncé par le gouvernement chinois cherche aujourd'hui à moderniser radicalement l'appareil de production chinois vieillissant pour en faire un centre d'innovation et de services.

Située dans la province méridionale du Guangdong, la ville de Shenzhen est une des pierres angulaires de ce projet. Centre névralgique de la production d'électronique dans le monde, Shenzhen a connu une transformation inédite; simple village au début des années quatre-vingt, elle est aujourd'hui l'une des mégalopoles les plus influentes du pays. Lors de sa visite en 1997, l'architecte et urbaniste Rem Koolhas s'étonnait d'y trouver l'« incroyable énergie spéculative du système communiste » qui construisait alors « des autoroutes qui ne touchent quasiment pas le sol » (Koolhas 1997). Vingt ans plus tard, la ville compte près de 10 millions d'habitants et sa fusion annoncée avec sa voisine Hong Kong pourrait en faire la plus grande région urbaine du monde. 
Fleuron industriel chinois, l'émergence de cette cité s'est faite sur l'impulsion d'une « zone économique spéciale » (ZES), attirant des géants de l'industrie mondiale, notamment taïwanais dont le géant Foxconn qui produit et assemble aujourd'hui les machines Apple et Samsung (Qiu 2014).

En marge de l'activité industrielle classique s'est développé dans la ville un vaste réseau d'entreprises spécialisées dans la fabrication de produits électroniques peu chers et sans marques, dits whiteboxes (Shih et al. 2010). Ces petites usines spécialisées se sont peu à peu spécialisées dans les copies de modèles existants à moindre coût, puis dans la production de designs originaux de basse qualité appelés en chinois shanzhai (Renaud 2015). Le tissu industriel urbain ainsi formé constitue aujourd'hui une infrastructure de production unique pour les objets électroniques de toutes sortes.

Une autre façon d'envisager les processus de fabrication (le maker movement) a dans le même temps émergé à différents endroits du monde (Anderson 2014, Lallement 2015). Petites séries, travail en flux tendu, réutilisation de design existants, les pratiques industrielles de fabrication chinoise du shanzhai trouvent un écho intéressant dans celle des makers. Fascinés, ils viennent du monde entier pour découvrir Shenzhen et apprendre à travailler en lien direct avec les usines (Lindtner, Greenspan \& Li 2015). L'open source joue un rôle central dans cette rencontre, garantissant à la fois la qualité des produits et une culture commune des processus de collaboration (Fernandez, Puel \& Renaud 2016). La ville de Shenzhen et ses environs offrent donc un terrain incroyablement riche pour observer les transformations à l'œuvre dans le monde de la fabrication et s'interroger sur leur rôle dans les processus de conception et d'innovation technologique.

\section{Méthodologie pour l'étude du selfie stick}

Si le shanzhai a été théorisé comme modèle d'innovation (IDEO 2012) ou d'entreprenariat (Zhao 2014), on trouve finalement assez peu d'articles décrivant précisément le fonctionnement de toutes ces petites usines. L'accès au terrain est assez difficile, car les groupes qui s'inscrivent dans ce réseau fonctionnent souvent dans l'illégalité. Le shanzhai, qui a trouvé ces lettres de noblesse dans la littérature scientifique étrangère, et plus récemment une reconnaissance au sein d'institutions officielles (Zhang \& Fung 2013), reste donc un sujet peu documenté, bien que très commenté. Je me suis largement heurté à cet obstacle pendant mon terrain. S'il est facile d'acheter des objets parfois loufoques, il est beaucoup plus compliqué de se rendre dans les usines, ou tout simplement de rencontrer les personnes qui travaillent dans ce milieu extralégal.

Le présent travail s'appuie sur plus de six années d'observation participante menée depuis 2010 dans le monde des technologies et de la fabrication en Chine. Plusieurs séjours dans la ville de Shenzhen au cours de l'année 2014 puis 2015 m’ont permis de conduire en chinois des entretiens auprès de vendeurs et producteurs de selfie sticks, dont certains possédaient des chaînes d'assemblages dans la région. J'ai également constitué un corpus d'images, d'entretiens préexistants et d'objets, en me concentrant sur les dynamiques et méthodes de fabrication des perches à selfie. 
La plupart des entretiens avec des vendeurs ont eu lieu au sein du quartier de Huaqiangbei à Shenzhen où se déroulent habituellement la vente et l'export de nombreux composants et accessoires électroniques. Les rencontres avec les responsables d'usines ont eu lieu principalement dans des usines situées dans le district de Bao An. J'ai réuni des éléments de documentation à partir de différents sites recommandés par les professionnels que j'ai interviewés, afin de mieux comprendre comment se déroulaient les achats. J'ai moi-même acquis divers modèles de selfie stick pour mieux les comparer.

\section{Résultats : les mécanismes de production du selfie stick}

La description la plus simple des modèles de fabrication des selfie sticks m’a sans doute été offerte par Nik, un exportateur d'électronique indien installé dans le quartier de Huaqiangbei, et à Shenzhen depuis une dizaine d'années.

«Quand un nouveau design commence à se vendre, les fabriquants shanzhai vont dans le magasin et en achètent une ou deux pièces. Ils le ramènent chez eux et le démontent entièrement pour voir comment il est fait. Pour chaque pièce, ils regardent comment ils peuvent le faire fabriquer pour moins cher. Certains ont de la famille qui font des caoutchoucs ou des boîtiers plastiques, certains peuvent avoir un meilleur prix sur les puces Bluetooth, certains vont essayer d'utiliser des éléments recyclés, d'autres enfin peuvent juste assembler plus rapidement. Il y a un incroyable rush pour envoyer le même modèle sur le marché au moindre coût donc les prix diminuent énormément pendant les deux premières semaines. »

La pratique du démontage / étude / assemblage semble être le premier ressort de ce type de conception lié à la fabrication. Il s'agit dans un premier temps d'identifier l'ensemble des composants afin de déterminer le ou lesquels pourront être substitués par un modèle moins cher ou proposant une fonctionnalité différente, afin notamment de se différencier sur un marché hypercompétitif.

La compétition sur le prix, mais également sur la vitesse d'exécution (time-to-market) exige une prise de risque maximale de la part des entrepreneurs du domaine. Les marges, essentiellement liées à la baisse des coûts de fabrication, sont très faibles (ici, quelques centimes par pièce seulement) et nécessitent donc de s'assurer d'être le premier et le moins cher pour ne pas finir avec des dizaines de milliers de pièces bloquées sur les étagères. "Dans le monde du shanzhai, les deadlines veulent vraiment dire que vous êtes morts ${ }^{8} »$, m'explique le fondateur de la marque Mixtile qui produit de petits ordinateurs open source à Shenzhen.

Cette baisse continuelle des coûts et les changements permanents de spécifications entraînent une grande instabilité des produits. D’après M. Jie qui travaille dans le marché de gros des accessoires à Huaqiangbei : « Les principaux acheteurs de selfie sticks sont les Coréens. Les Brésiliens adorent ça aussi récemment, surtout les modèles pour iPhone ». 
2ab. Plusieurs produits sont réalisés par des constructeurs distincts et possèdent pourtant le même numéro de modèle.

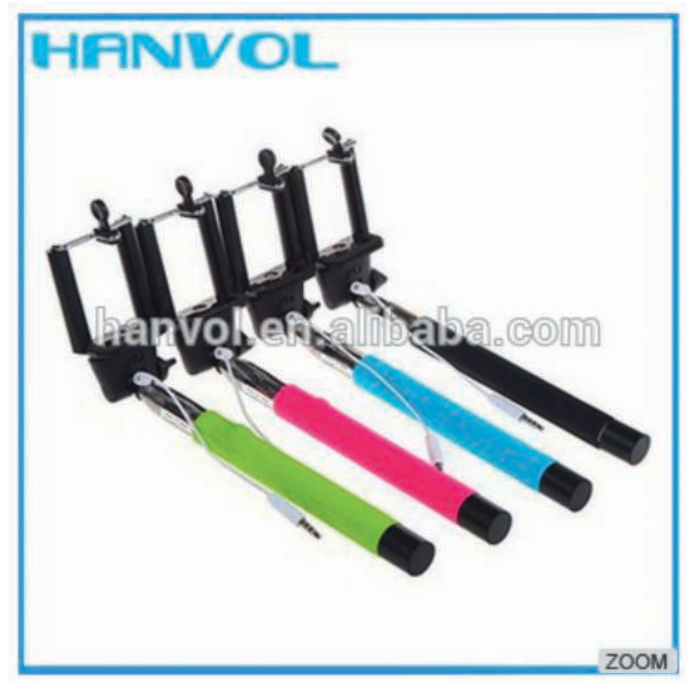

Wired Selfie Stick Handheld Monopod Z07-5S selfie stick Cellphone Holder for Apple iPhone Samsung Android

FOB Reference Price : Get Latest Price

US \$0.8-1.5/Piece 100 Piece/Pieces selfie (Min. Order)

Supply Ability. 10000 Piece/Pieces per Day selfie stick

Port: Shenzhen Port

$\square$ Contact Supplier

M. Hu, un autre vendeur situé dans le SEG Electronic Market, propose un des modèles les moins chers (6.5 RMB, soit 1 euro environ), qu'il affirme avoir produit dans une usine tenue par un membre de sa famille près de la ville de Zhuhai, plus loin dans le Delta de la Rivière des Perles. Le modèle est constitué d'un simple tube télescopique d'aluminium avec une poignée en plastique et une pince pour mettre un téléphone. Son fils, qui travaille avec lui dans la boutique m'explique : « ce modèle date d'il y a environ un an. Le produit a largement évolué depuis, et maintenant, nous avons un modèle avec Bluetooth. Beaucoup de téléphones ne supportent pas le Bluetooth donc nous avons aussi celui-ci avec un cordon. C'est mon préféré car il épargne la durée de vie de la batterie ». D’autres évolutions du selfie stick proposent une tête rotative ou un flash intégré.

Le modèle le plus commun lors de ma première visite à Huaqiangbei semble être numéroté Z07-5S. Pourtant, en regardant de plus près, ce numéro recouvre une foule de modèles aux fonctionnalités diverses, dédiés à des marques de téléphones également disparates et qui plus est produits par différents constructeurs.

Pourtant, d'après mes observations, les vendeurs sont capables de reconnaître un modèle et d'en donner approximativement l'âge (en mois) et la provenance (usine ou revendeur) - si tant est qu'il s'agisse d'un modèle répandu. La boîte, qui indique pourtant le numéro du produit, n'est pas utile pour le reconnaître. Comment se fait-il que des dizaines de produits possèdent tous le même numéro identifiant ? Une simple recherche sur le site 1668 du groupe Alibaba permet d'imaginer une première réponse : la boîte portant ce numéro est simplement la moins chère du site.

Les différents sites de la firme d'e-commerce chinoise Alibaba ont joué un rôle extrêmement important dans la diffusion des produits chinois depuis 20 ans : le site alibaba.com 
a servi d'articulation entre les producteurs chinois et les acheteurs du monde entier ; les sites Taoabo puis Tmall servent aujourd'hui d'infrastructure au boom de la consommation en Chine ; AliExpress permet désormais la vente directe depuis la Chine aux particuliers du monde entier; enfin le site 1688.com permet aux usines chinoises d'acheter en gros des pièces ou des produits finis.

Le site 1688, nommé ainsi car la lecture des chiffres en chinois sonne comme le mot Alibaba, est un des centres névralgiques de l'appareil productif chinois. Une visite approfondie de ce site permet de mieux comprendre comment il est possible de se lancer dans l'assemblage de selfie sticks. Il suffit d'écrire les requêtes « emballage de selfie sticks » dans la barre de recherche pour voir apparaître des centaines de modèles différents. À la date de cette recherche, la moins chère coûtait RMB 0.30 (USD 0.05) par unité pour plus de 1000 achetés - avec un prix dégressif pour 10 000, 100 000, etc. Le vendeur annonce sur le site avoir des millions d'unités en stock. Sans effectuer d'autres requêtes, nous serions capables de réunir la quasi-totalité des pièces nécessaires pour construire des perches. Le système de recommandation propose d'ores et déjà les poignées et d'autres pièces comme notamment les circuits imprimés et les cordons. Pour les tubes en aluminium, une interface dédiée permet de rentrer la taille, le diamètre et la qualité puis de comparer les prix par constructeur. Ces pièces sur mesure pourraient être livrées en deux jours à notre porte. Là encore, des millions d'unités sont annoncées en stock.
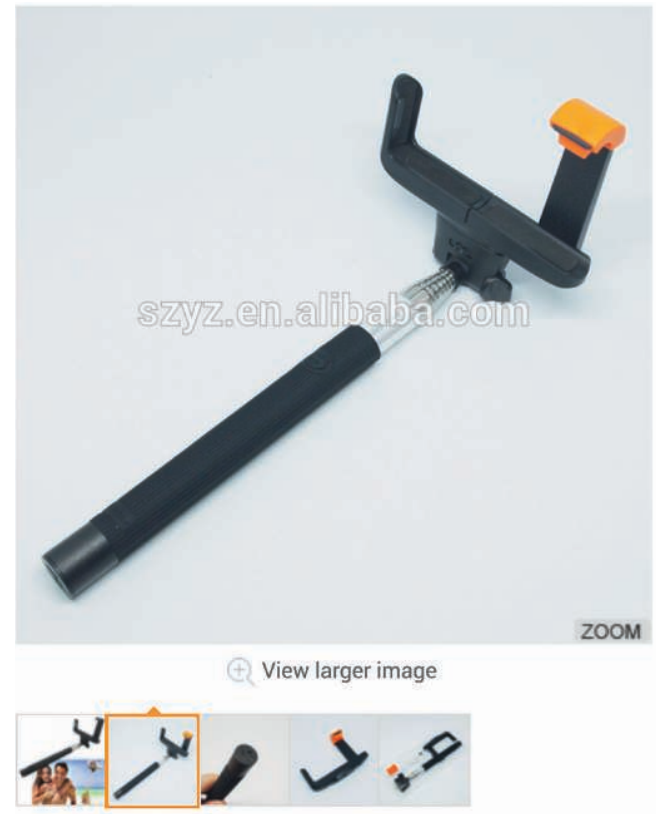

\section{Wireless Bluetooth Z07-5s Monopod Selfie Stick for samsung self charge}

Free Inspection

FOB Reference Price : Get Latest Price

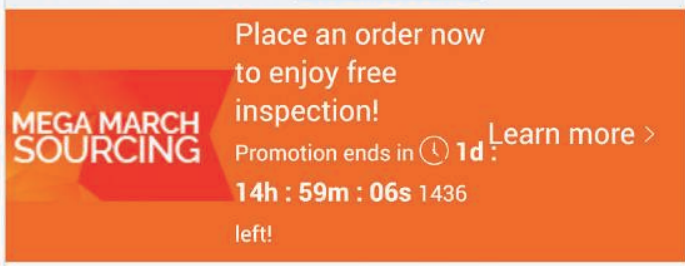

US \$4.29-6.56

(Min. Order)

Supply Ability. 10000 Piece/Pieces per Day

Port: Shenzhen/HK

Contact Supplier 
3. Le site 1688 d'Alibaba fait du sourcing (de «l'approvisionnement ») de pièces un jeu d'enfant.

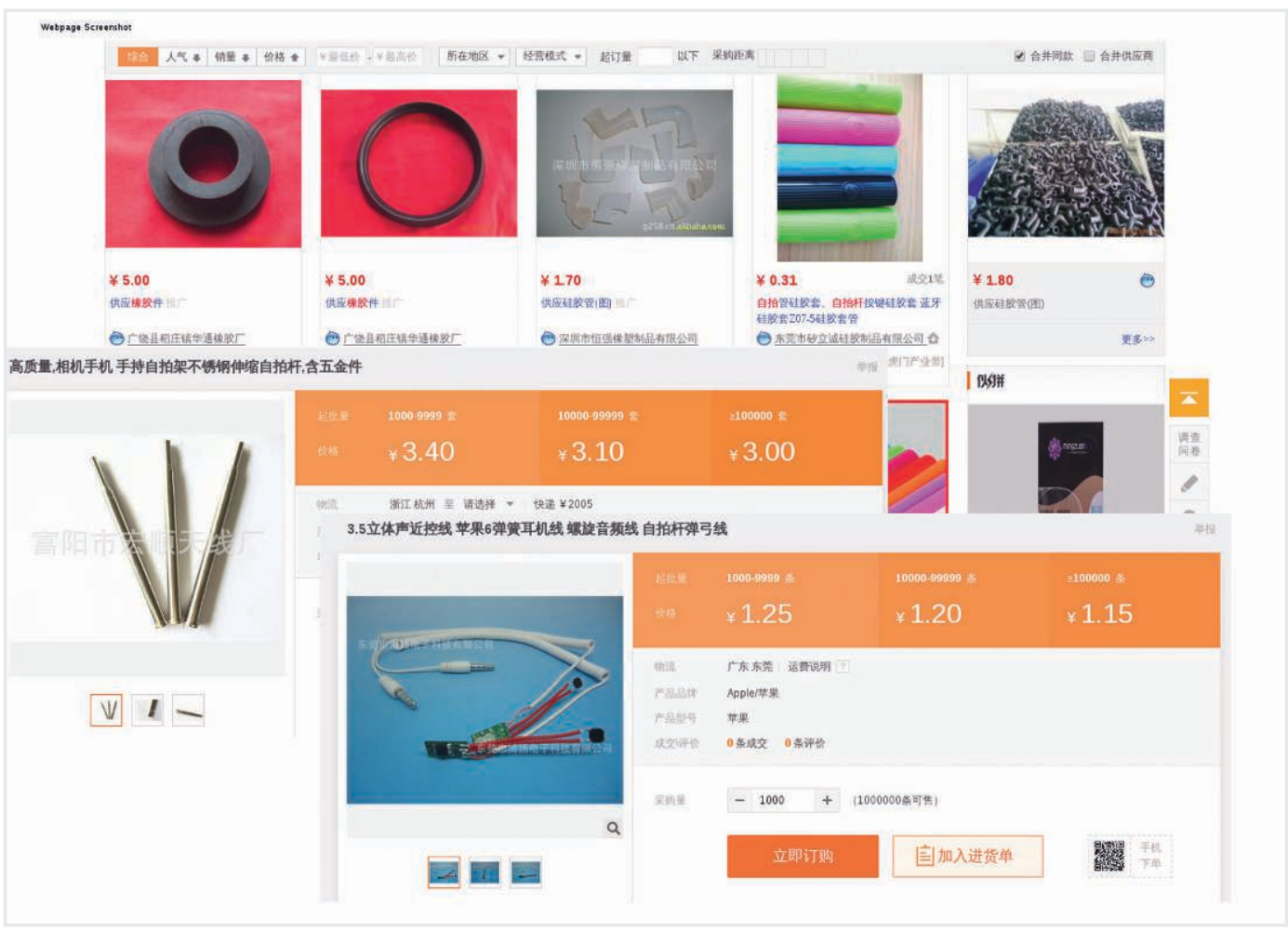

Bien sûr, la complexité du processus de fabrication ne saurait être minimisée. L'étape de l'assemblage apparaît notamment comme primordiale. Les conditions de travail dans les cités usines de la région sont - tristement - célèbres. Les migrations importantes depuis les campagnes ont amené énormément de femmes et d'hommes dans cette région où les besoins en main-d'œuvre ont longtemps paru impossibles à satisfaire tant la demande était grande (Qiu op. cit.). Les usines affichent donc sur leurs sites de nombreux certificats concernant la qualité des conditions de travail de leurs employés et la propreté de leurs installations. Le système, supervisé par le gouvernement, repose sur une page web censée faire foi. Les photos des employés au travail font figure d'argument, comme dans le cas de cette petite entreprise spécialisée dans les câbles électroniques (figure suivante).

Les usines d'assemblage sont situées souvent en banlieue de Shenzhen, notamment dans le district de Baoan, mais également plus loin dans le delta (Al 2012). Cette proximité, d'abord géographique, entre assemblage et production, est accrue par la présence d'une infrastructure numérique importante qui permet une communication et une livraison rapide de pièces dans tout le delta - et plus largement en Chine.

Le gouvernement chinois subventionne l'envoi et le transport de marchandises simultanément en Chine et vers l'étranger, soutenant ainsi le développement de relations toujours plus 
étroites au sein de ce réseau d'entreprises, de producteurs et de vendeurs dans la région. Le cadre fiscal pour ces usines est aussi grandement simplifié par la classification de Shenzhen comme zone économique spéciale depuis les années quatre-vingt, et il a été régulièrement revu et étendu aux régions annexes autour de Shenzhen. La relative frilosité du gouvernement à contrôler et taxer ces petites usines permet aux vendeurs de gérer leurs comptes très librement. Plusieurs vendeurs interrogés affirment ne pas tenir de comptabilité à long terme, mais uniquement un relevé de leurs activités afin de gérer les flux d'argent dans leur entreprise. L'intervention du gouvernement dans la construction de cet écosystème particulier de production est donc importante, tant dans le subventionnement que dans la définition de règles de gestion adaptées.

\section{Discussions : l'assemblage comme modèle de conception par la fabrication}

Le cas du selfie stick permet donc de voir comment se constitue dans la région de Shenzhen un appareil de production sous la forme d'un réseau dense, à la fois numérique et géographique. Une des grandes forces de ce système consiste en effet à offrir un accès à une grande quantité de pièces existantes permettant une recombinaison rapide pour construire de nouveaux objets, produits ou services. Cette pratique de l'itération rappelle par bien des aspects les modèles que l'on associe à la notion d'innovation ouverte, à ceci près que les biens échangés ne sont pas immatériels (connaissances ou savoir-faire), mais des objets réels. La fluidité des échanges de biens tangibles permet l'accumulation de connaissances quant aux ressources disponibles, et donc d'envisager leur recombinaison sous des formes nouvelles. L'étape du design est conçue comme une étape préliminaire dont le but est de déterminer la capacité à fabriquer. La fabrication préside donc ici à la conception. Il ne s'agit pas de modifier différents plans ou blueprints (dessins de définition) existants, mais bien directement d'assembler des parties de l'objet à fabriquer qui auront déjà été conçues par d'autres pour créer une nouveauté.

La recomposition permanente des produits sous un même nom et la disparition des numéros de produits vers une succession de versions montrent à quel point les itérations peuvent être rapides, sous l'influence du marché comme premier déterminant. Au contact des chaînes de fabrication, le travail de conception donne lieu à des assemblages inédits qui dépendent directement des ressources et technologies disponibles. L'assemblage d'objets à la fois électroniques (une puce Bluetooth) ou autres (une poignée en plastique ou un tube en aluminium), invite à redéfinir l'idée même que l'on peut se faire d'un objet technologique. Finalement, c'est peut-être le site 1688.com qui représente la véritable clé d'un tel processus dans la mesure où il permet une synchronisation des différents acteurs et procédés. La conception ne s'entend donc plus nécessairement comme un acte d'imagination créatrice, 
mais plutôt comme un travail de composition qui aboutit à reconfigurer des éléments existants sous une forme plus ou moins novatrice. Ce processus de recomposition se déroule de manière progressive et itérative, au fil de l'apprentissage des usages possibles, des ressources disponibles et des pratiques d'assemblage. Ce dernier apparaît ici comme une pratique centrale, bien qu'historiquement peu considéré dans les processus de design.

Si le shanzhai a accédé à un statut presque mythique, ses participants n'en sortent pas toujours indemnes. Chez ces entrepreneurs, rares sont ceux qui peuvent se vanter d'avoir réussi, c'està-dire de s'être enrichis suffisamment pour faire accéder leur activité à un statut qui soit, tout à la fois, rentable et légal. L'annulation dans les faits du cadre légal soumet l'ensemble des participants de ces réseaux de fabrication à une compétition dérégulée. La fluidité et la vitesse tant révérées dans le monde du shanzhai ne peuvent exister que grâce à des réseaux de confiance, liés notamment à l'implication de familles entières dans ces processus. Les risques de cette compétition s'appliquent non seulement aux entreprises, mais également à leur personnel et à l'environnement tout entier - du fait des méthodes employées ou encore de l'usage fréquent de produits souvent toxiques. Une approche de la définition du produit, radicalement définie en termes de marché de destination fait souvent peu de cas des employés ou des conséquences sur l'environnement, ni même, véritablement, en fin de compte, de ses réels usagers.

4. L'assemblage des cordons mini-jack pour selfie stick

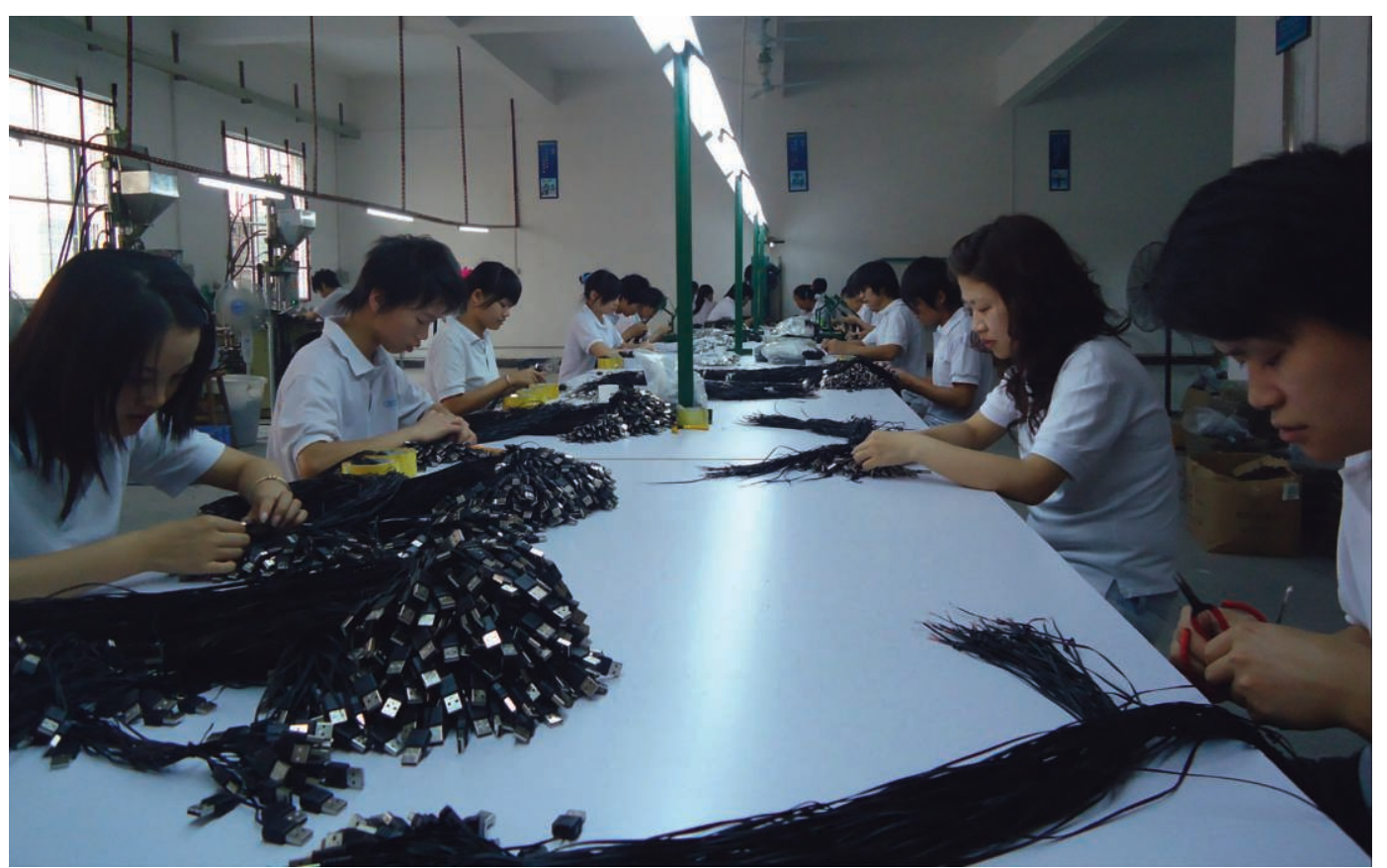


L'idée même d'une innovation complètement centrée sur la fabrication paraît sortir d'un rêve d'industriel fou. Plus de conception ou de nouveautés, l'intégralité des ressources peut enfin être dédiée à la production - et à la consommation. Repris dans une large mesure par les makers du monde entier, ce modèle n'est pas sans risque car il pose entre autres la question de la régulation d'un tel réseau de producteurs, et bien sûr de la propriété des appareils de production. L'enrichissement direct de certaines parties prenantes de cet écosystème ne saurait cacher les nombreux laissés pour compte d'un modèle industriel du shanzhai supposé servir d'exemple. Le modèle d'une innovation technologique centrée sur l'assemblage et la fabrication promet des changements profonds dans les modes de production industrielle, et donc dans nos façons d'être au monde, de se le représenter et d'en partager la connaissance. Néanmoins, il semble primordial de construire dans le même temps de nouvelles formes de régulation de ces réseaux émergents qui soient capables, en particulier, d'éviter un immense gâchis - celui du transfert, après seulement quelques jours à peine d'utilisation, de dizaine de milliers de selfie sticks de l'usine à la poubelle. 


\section{Notes}

1. Is This the World's Oldest Selfie Stick? In NY Mag: nymag.com/selectall/2016/04/is-this-the-worldsoldest-selfie-stick.html.

2. «If only Bradley's arm was longer. Best photo ever. \#oscars", Twitter: twitter.com/theellenshow/ status/440322224407314432.

3. Bloomberg: bloomberg.com/news/articles/2014-12 31/selfie-sticks-rule-holiday-season-as-musthave -accessory.

4. CityAM : cityam.com/206530/selfie-stick-short-history-years-most-popular-christmas-present.
5. Pour une liste plus complète, se référer à la liste «List of selfie-related injuries and deaths » maintenue sur Wikipedia: en.wikipedia.org/wiki/ List_of_selfie-related_injuries_and_deaths.

6. BBC: bbc.com/news/uk-england-london-30719592.

7. Mashable: mashable.com/2015/02/17/aus tralia-galleries-selfie-stick.

8. Citation originale: «In the shanzhai business, deadlines really mean you are dead. »

\section{I'auteur}

Clément Renaud est chercheur et artiste. Il s'intéresse à la production d'espaces et de représentations par les technologies. Spécialiste de l'innovation en Chine, il est aujourd'hui membre de l'Institute for Area and Global Studies à l’École Polytechnique Fédérale de Lausanne.

\section{Iconographie}

Image d'ouverture. Panneau d'interdiction du selfie stick dans le musée de Brisbane. (C) Kerry Raymond - CC BY 4.0, d'après Wikipedia.

\section{Références}

Al, S. dir. 2012 Factory Towns of South China. Hong Kong: HK University Press.

Anderson, C. 2012 Makers: The New Industrial Revolution. Danvers: Crown Business.

Bruno, N., Bertamini, M., Protti, F., Humphrey, N. et al. 2015 «Selfie and the City: A World-Wide, Large, and Ecologically Valid Database Reveals a Two-Pronged Side Bias in Naïve Self-Portraits », PLOS ONE, 10 (4). DOI: 10.1371/journal.pone.0124999.

Chesbrough, H. W. 2003 «A Better Way to Innovate», Harvard Business Review (July).

Edgerton, D. 1998 «De l'innovation aux usages. Dix thèses éclectiques sur l'histoire des techniques », Annales. Histoire, Sciences Sociales 53 (4) : 815-837. DOI: 10.3406/ahess.1998.279700.

Fernandez, V., Puel, G., \& C. Renaud 2016 «The Open Innovation Paradigm: from Outsourcing to Opensourcing in Shenzhen, China», International Review for Spatial Planning and Sustainable Development 4 (4): 27-41. DOI: doi.org/10.14246/irspsd.4.4_27.
1. Clément Renaud - CCO.

2 a $\&$ b. Alibaba.com

3 \& 4. 1688.com / Alibaba.

IDEO 2012 Shanzhai | Patterns from IDEO. Retrieved May 31, 2013, from http://patterns.ideo.com/issue/ shanzhai/

Kawakami, K. 2005101 Unuseless Japanese Inventions in H. Fearnley-Whittingstall dir. New York: W. W. Norton $\&$ Company

Koolhaas, R. \& the Harvard Project on the City 2000 in Mutations. Bordeaux / Barcelone: arc en rêve / Actar.

Lallement, M. 2015 L'Âge du faire. Hacking, travail, anarchie Paris: Seuil (La Couleur des idées).

Lecoq-Ramond, S., Paccoud, S., Schäfer, D. 2016 Autoportraits: de Rembrandt au selfie. Staatliche Kunsthalle Karlsruhe / Musée des Beaux-Arts de Lyon / National Galleries of Scotland.

Lindtner, S., Greenspan, A. \& D. Li 2015 « Designed in Shenzhen: Shanzhai Manufacturing and Maker Entrepreneurs », Aarhus Series on Human Centered Computing 1 (1): 85-96. http://doi.org/10.7146/aahcc. vlil.21265. 
Morozov, E. 2014 To Save Everything, Click Here. The Folly of Technological Solutionism. New York: PublicAffairs. http://doi.org/10.1007/s13398-014-0173-7.2.

Ong, W. 2002 Orality and Literacy: The Technologizing of the Word. London: Routledge.

Qiu, J. L. 2014 «Farewell iSlave: Foxconn, Digital Capitalism, and Networked Labor Resistance» Society 34 (4) : 119-137.

Renaud, C. 2015 «Bandits brought Technology to this World» in N. Maigret \& M. Roszkowska dir. The Pirate Book. Ljubljana: Aksioma.

Rettberg, J. W. 2014 Seeing ourselves through technology : how we use selfies, blogs and wearable devices to see and shape ourselves. Basingstoke: Palgrave Macmillan. DOI: 10.1057/9781137476661.

Simonsen, J. \& T. Robertson 2013 Routledge international handbook of participatory design. London: Routledge.

Stiegler, B. 2008 Réenchanter le monde: La valeur esprit contre le populisme industriel. Paris: Flammarion.
Ueda, H. \&Y. Mima 1985 Telescopic Extender for Supporting Compact Camera. US 453058A. Google Patents. http:// www.google.com/patents/US4530580.

von Hippel, E. 2006 Democratizing Innovation. Cambridge: The MIT Press. En ligne: https://www.dropbox.com/s/ r4079o59kciewod/2333.pdf?dl=0.

Shih, W.C, Chien, C.-F. \& J.-C. Wang 2010 «Shanzai! Mediatek and the "White Box" Handset Market " Harvard Business School Technology \& Operations Mgt. Unit Case No. 610-081.

Zhang, L. \& A. Fung 2013 "The Myth of "Shanzhai" Culture and the Paradox of Digital Democracy in China », Inter-Asia Cultural Studies 14: 401-416. Doi: 10.1080/14649373.2013.801608.

Zhao, J. 2014 «Shanzhai: An Emerging Entrepreneurial Model », Management and Administrative Sciences Review 808: 762-808

\section{Pour citer l'article}

Renaud, C. 2017 «L'assemblage comme forme d'innovation: la fabrication d'un selfie stick à Shenzhen », TechniquesECulture 67 « Low tech? Wild tech!», p. 100-115. 